\title{
Best Papers Selected at the 14th Eurographics Workshop on Graphics and Cultural Heritage Editorial
}

The Eurographics Workshop on Graphics and Cultural Heritage is the premier venue of the Eurographics (EG) Working Group on Graphics and Cultural Heritage, whose aim is to promote the discussion and exchange of information between researchers on visual media technologies and cultural heritage.

Initially, the focus of the Graphics and Cultural Heritage (GCH) community has been the introduction of new technologies driven by computer scientists and the appropriation of these technologies by human scientists and cultural heritage $(\mathrm{CH})$ scientists. Today, the first results of a genuine cross-fertilization may be appreciated: on one hand, archaeologists, architects, conservation scientists, and curators foster the use of digital tools within their everyday study of $\mathrm{CH}$, and consequently renew their methodological approaches; on the other hand, computer scientists propose computational methods not only to acquire more and more accurate digital data but also to extract relevant information for the analysis and interpretation of cultural assets.

The 14th edition of the GCH workshop took place in Genova, Italy, October 5-7, 2016. The scientific program of GCH 2016 is a good portrait of such a multidisciplinary scientific community looking at innovative paths for bridging the gap between $\mathrm{CH}$ documentation processes and digital processing. Indeed, we have particularly encouraged interdisciplinary approaches targeting analysis, classification, and interpretation to explore the true potential of deploying Information and Communication Technologies (ICT) for advancing knowledge in the $\mathrm{CH}$ domain. We compiled an exciting program with 29 technical papers, 8 international project papers, 3 keynotes, a special session on 3D mass digitization, and a panel session to discuss future research challenges. More than 70 scientists actively participated in the symposium, raising lively discussions in the various sessions.

We received 41 submissions of scientific contributions from several countries in the world, which have been revised by the 46 members of the program committee. A first selection of eight candidates for Best Paper were identified based on the reviews, and a special committee of five members was set up for the final award of the best papers at the conference venue. The evaluation process measured both the degree of innovation and the interdisciplinary value, assessing how much the Computer Graphics (CG) and $\mathrm{CH}$ aspects were intertwined together. In the end, two papers were chosen, which were able to fulfill both criteria. Those papers were invited to this special issue of ACM fournal on Computing and Cultural Heritage, and cover two challenging research directions in the field.

The first paper by Agus et al., "Data-Driven Analysis of Virtual 3D Exploration of a Large Sculpture Collection in Real-World Museum Exhibitions," addresses the problem of visual and interactive communication of digital artifacts. It discusses an extensive data-driven analysis of usage experience based on logs gathered in a two-year period at four exhibitions in archeological museums. The main trends in visitor behavior during the interactive sessions were highlighted, providing useful insights for the design of 3D exploration user interfaces in future digital installations.

The second paper by Sizikova and Funkhouser, "Wall Painting Reconstruction Using a Genetic Algorithm," fits the analysis and interpretation of cultural assets. In particular, it tackles the problem of the reconstruction of frescos from fragments. Due to the presence of eroded and missing pieces, digital reconstruction is a complex problem. The authors propose an unsupervised genetic algorithm, which improves the quality of the partial reconstructed clusters with respect to previous approaches.

2017 Copyright is held by the owner/author(s).

1556-4673/2017/12-ART1

https://doi.org/10.1145/3152908 
We would like to thank the Event Chairs, Bianca Falcidieno and Dieter Fellner, the GCH dedicated committee for the best papers, the program committee and the reviewers, as well as the editor-in-chief of the ACM fournal on Computing and Cultural Heritage, Roberto Scopigno, for making this possible.

Chiara Eva Catalano

Livio De Luca

GCH 2016 Program Chairs 